\title{
Téoros
}

Revue de recherche en tourisme

\section{Du jeu au développement économique : technopoles et parcs thématiques}

\section{Yves Robillard}

Volume 12, numéro 3, octobre 1993

Le renouveau des parcs à thèmes

URI : https://id.erudit.org/iderudit/1077938ar

DOI : https://doi.org/10.7202/1077938ar

Aller au sommaire du numéro

Éditeur(s)

Université du Québec à Montréal

ISSN

0712-8657 (imprimé)

1923-2705 (numérique)

Découvrir la revue

Citer cet article

Robillard, Y. (1993). Du jeu au développement économique : technopoles et parcs thématiques. Téoros, 12(3), 39-42. https://doi.org/10.7202/1077938ar d'utilisation que vous pouvez consulter en ligne.

https://apropos.erudit.org/fr/usagers/politique-dutilisation/ 


\title{
Du jeu au développement économique : technopoles et parcs thématiques
}

\author{
Yves Robillard*
}

«Plus on joue fort, plus on pense fort! $m$ C'est Alvin Toffler ${ }^{(1)}$ qui parle. Il est à Silicone Valley et nous fait visiter les centres de conditionnementphysiqueinstallés à même les fabriques d'ordinateurs. On paie aux employés de tels services pour qu'ils donnent un meilleur rendement. *Des cerveauxau soleil: les entreprises de pointeont tendance à s'installer au soleil pour l'environnement deloisirs», écrit Anita Rudman dans le journal Le Monde ${ }^{(t)}$. J'ai devant les yeux la publicité du Sophia Country Club de Sophia-Antipolis, avec ses tennis, piscines, studios, salles de séminaires et *Club House conçu à l'image d'une ville antique romainew, etc. Il est évident que dans les technopoles, jeu et travail ne sont plus des valeurs qui s'opposent!

Le jeu opposé au travail, comme dans le conte de Pinnochio, est une invention de la société industrielle qui avait besoin de stimuler l'endurance des travailleurs à la chaîne. Dans les sociétés agraires, la plupart des travaux se font en chantant. Vous le pressentez: je suis un défenseur de l'apprentissage par le jeu. L'enfant, avant d'arriver à l'école, apprend tout par le jeu. Et après, il joue à l'école pour bien assimiler. L'enfant est totalement concentré dans ses jeux. Et chaque jeu a des règles!

\section{L'importance du jeu}

Dans les nouveaux types d'entreprises des technopoles, écrit Thierry Gaudin ${ }^{(5)}$, *ce qui est délicat, c'est la préparation des relations et des compétences nécessaires pour utiliser les nouveaux équipements à bon escient. L'accent est mis sur le «relationnels en tant que fondement de transformation et de solidarité au sein de l'entreprise. Les mutations tendent à promouvoir davantage de participation et d'initiative des salariés. Un nouveau style de management commence alors à être

Monsieur Yues Robillard est professeur au Dépar. tement d'histoire de l'art de I'UQAM. pratiqué. Les responsables deviennent des animateurs afin de stimuler à la fois la motivation collective et les motivations individuelles. Cette primauté du bien-être des employés s'accompagne de l'introduction de l'irrationnel dans l'entreprise ${ }^{(4)}$ par toutes sortes de wcentres de ressourcement: où se côtoient le meilleur et le pire». Et Gaudin en donne plusieurs exemples: les gens de chez Apple se ressourçant dans une hutte amérindienne, Bob Aubry faisant marcher sur de la braise les cadres de diverses entreprises. Il est question de \&lunettes cosmiques», du *Heavy Foetal» (dérivé des caissons d'isolation), de la visualisation positive, etc. Gaudin prédit qu'à ces «départements de ressources humaines", apparus dans les années 1980, succèderont des «cercles de connaissance humaines, venant d'une meilleure connaissance de la parapsychologie et des recherches sur le cerveau.

Les centresderessourcement danslesnouvelles entreprises sont capitaux. «Matière grise et pins parasols: Sophia-Antipolis, çà marche, mais chercheurs, enseignants, et industriels ne forment pas encore une communautés, c'est le titre d'un article de ClairedeNarbonneparuen $1985^{\circ}$. Leliant, c'est le jeu! L'interdisciplinarité réelle ne s'installe que par des expériences de vie communes. Les *groupes de croissances utilisent toutes sortes de jeux. Le jeu est ce qui soude une technopole. C'est le fertilisant del'avenir. L'attitude ludique est avant tout un état d'esprit. Apprendre à jouer d'un instrument de musique n'est pas facile, mais on le fait «par plaisirs. Et joserais même affirmer que c'est dans la mesure où une technopole assume bien sa fonction ludique qu'elle prépare bien l'avenir!

\section{Le «Project Sunrise»}

J'ai eu la chance de participer à l'élaboration d'un projet de parc thématique devant mener à la création d'une technopole. Ce parc conçu pour la revitalisation du VieuxPort de Sydney, le Darling Harbour, nevit pas le jour, mais il est intéressant de le comparer au Futuroscope de Poitiers pour voir le rapport entre théories et pratiques, aspirationset réalisations. Baptisé «Project Sunrises, ce parc a été élaboré de 1981 à 1985 et devait coûter $\$ 210$ millions australiens. En décembre 1984, on posait la première pierre du Futuroscope, ouvert en 1987, et l'investissement en 1989 se chiffrait dans les $\$ 200$ millions.

L'humanité est entrée dans une nouvelle phase d'évolution: les nouvelles technologies changent la face du monde! Elles peuvent être aliénantes ${ }^{(6)}$ ou libératrices. Familiariser les gens à ces nouvelles technologies, et trouver ensemble comment elles peuvent améliorer la qualité de vie de la majorité, tels étaient les buts $\mathrm{du}$ *Project Sunrises ou *Projet du Nouveau Soleil » ou $*$ du Soleillevant $»$. Le *Project Sunrise» était un parc urbain à proximité du centreville, dont les bâtiments étaient sous-terre ou en hauteur de façon à dégager les espaces verts et la vue sur les berges. Il était constitué des 6 pavillons du *Parc de la Découverte*, de la *Communauté expérimentale du Pyrmont Bridgew, de ol'Institut des Technologies Sunrises, d'un *Centre des Congrès», d'un *Centre d'Exposition», d'un complexe hôtelier, d'espaces à bureaux et développement domiciliaire pour la future technopole, et d'un marché.

\section{Le Parc de la Découverte}

Dans les 6 pavillons du parc thématique, le visiteur était invité à se familiariser avec les technologies Sunrise, en manipulant celles-ci, et participant activement à une situation dramatiqueimpliquantuneréponse de la part du groupe dans lequel il se trouvait. Des programmes pour débutants et personnes plus averties étaient disponibles. Il était aussi possible de se faire commenter le travail de spécialistes que l'on observait.

Sur le plan de l'attrait touristique, chaque pavillon évoquait un site important ou lieu mythique de l'Australie, le Barrier Reef (Barrier Reef Aquarium), le Ayers Rock (Mineral Mountain et Energy Rock), les 


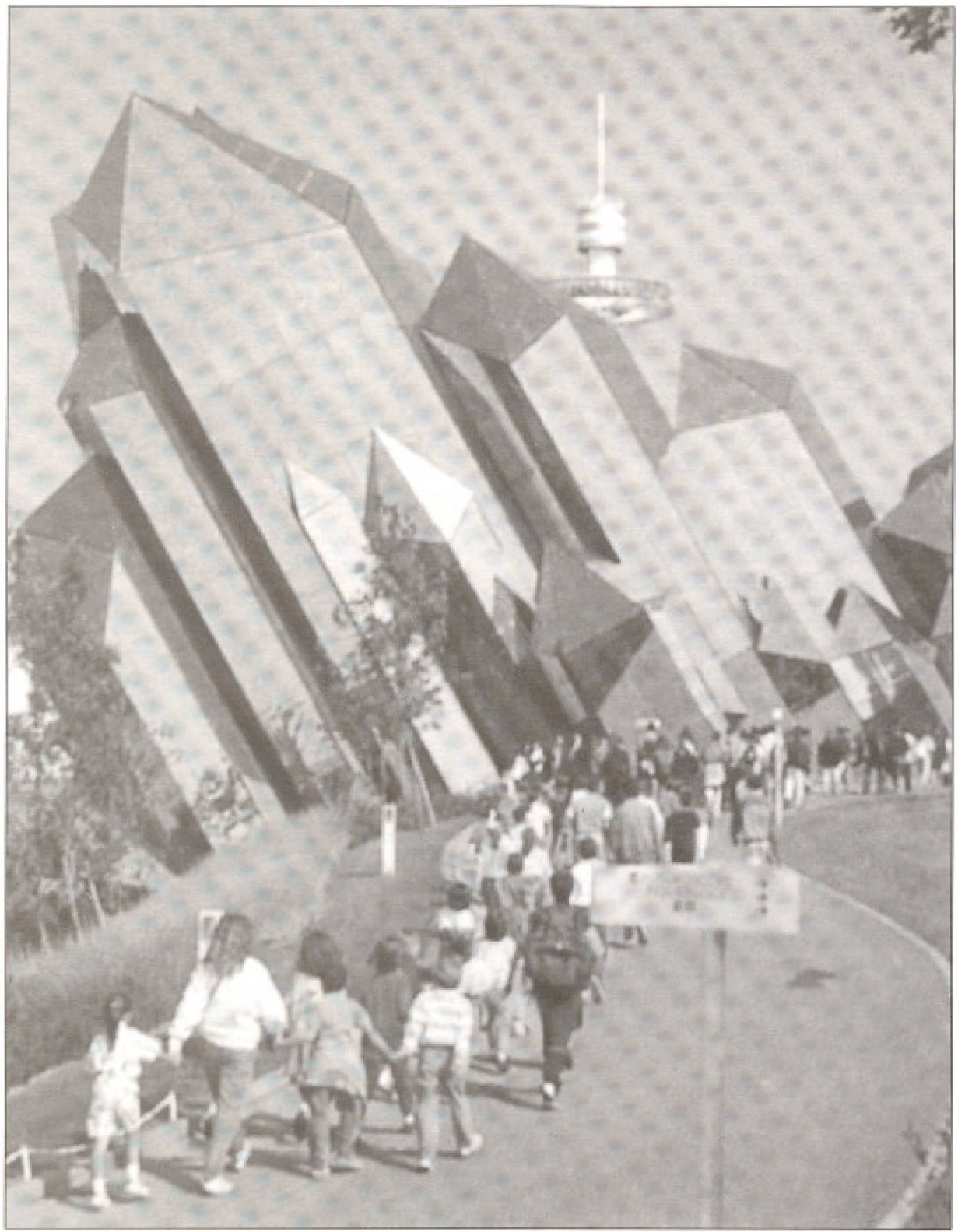

cavernes arborigènes (Dreamtime), les grandes steppes désertiques (Green Desert), leciel de la Croix du Sud (The Orbisphere) et les espèces de fauneet de flore anciennes et actuelles de l'Australie (Ancient Australia).

Dans chaque pavillon, on démontrait l'application decertaines technologiesde pointe à un domaine particulier, en questionnant les répercussions socio-culturelles. Exemple: le *Barrier Reef Aquarium» était constitué de 3 immenses citernes dans lesquelles on se promenait en sous-marins, permettant de voir poissons, récifs et coraux. On arrivait à un laboratoire d'où il fallait diriger les robots affectés à la mariculture, comprendre les programmes les
Community of Tomorrown, de 1000 habitants y vivant jour et nuit. Cette communauté électronique aurait eu comme fonction d'adapter continuellement son modedevieauxnouveaux développements technologiques. Elle devait s'auto-suffire et demeurer très consciente de la nécessité de toujours équilibrer le bien-être matériel et spirituel de ses membres.

Les habitants devant déterminer leur propre mode de vie, il est impossible de le décrire. On peut le supposer! L'architecture modulaire, transformable électroniquement, aurait été à la fois lieux de travail, d'instruction, de repos ou de loisirs. L'expertise de la communauté étant dans les domainesdel'informatique, de la robotique, de la télématique, on peut supposer qu'ily aurait eu là-dessus de nombreux centres de formation et banques de données, mais aussi des cafés, restaurants, boutiques, centres de création en design, audiovisuel, et jeux divers. Et le soir, des discothèques nouveaux genres seraient apparues, tout comme de nouveaux *centres de ressourcements ou «groupes de croissances, propres aux «Communautés de l'Aubes:?

\section{Le rôle de l'Institut}

*L'Tnstitutdes Technologies Sunrise»était ce qui devait faire le lien entre le parc et le pont, le grand public et les entreprises. Les anglophones sont pragmatiques. La premiêre tâche de l'Institut allait être de construire le parc et le pont et d'y créer une communauté. On imaginait pour cela l'organisation de séminaires impliquant des visionnaires de renom international pour donner les grandes lignes de directions de recherche, et plusieurs groupes de travail s'occupant de la promotion des industries hi-tech australiennes et des divers scénarios pour le parc et le pont.

L'Institut était vue comme la source de création des idées réalisées dans le parc et sur le pont. On le considérait comme un centrespécialisé dans l'apprentissage par le jeu, etl'application des technologies Sunrise aux loisirs éducatifs, et à la vie courante dans le laboratoire vivant de la communauté du pont. Dans une seconde phase, après l'ouverture du parc, l'Institut donnerait des cours et séminaires sur ces sujets, et se consacrerait à l'éducation populaire, soucieuse de rejoindre les délaissés. Les gens du *Project Sunrise* disaient: *Nous 
pensons que l'Institut verra se créer autour d'elle un quartier où toutes les compagnies hi-tech voudront avoir leurs bureaux, générant ainsi un nouveau Silicone Valley. Le *Project Sunrises, financièrement appuyé, n'a pas vu le jour pour raisons politiques.

\section{Le Futuroscope}

C'est la volonté politique qui està l'origine du Futuroscope, celle de René Monory, ancien ministre de $\mathrm{l}^{\prime} \mathrm{E}$ ducation nationale et président du Conseil général de la Vienne. Au début, en septembre 1983, il ne s'agissait que de créer une avitrine technologiques, montrant que les gens du Poitou s'intéressaient aux technologies de pointe. Ce fût de bâtiment du *Futuroscope» ouvert en mai 1987 (un prisme surmonté d'une sphère), \&le soleil se levant sur un monde en mutations», comme le décrivait son architecte Denis Laming. Mais très tôt, le 4 février 1985, le Conseil de la Vienne acceptait que ale pavillon du Futuroscope soit compris dans un ensembleplus vaste, constituéd'une zoneludique, d'une aire de formation et d'une aire d'activités technologiques».

Pourquoi le Futuroscope? *Parce que l'opinion, dit René Monory ${ }^{(b)}$ accepte avec plus ou moins de résistance les nouvelles technologies. Nous vivons dans une société de mutations rapides et incontournables. Il faut donc mettre en contact le public avec ces technologies nouvelles, les sensibiliser à leur caractère inéluctable, les banaliser... dédramatiser le futur», dira$\mathrm{t}$-il ailleurs. Ainsi donc la philosophie du Futuroscopesera-t-elled'établir uneétroite synergie entre loisirs, formation, et production, activitésréunies autour d'un thème unique, le traitement de l'information.

\section{Le Parc européen de I'Image}

Au début, on imaginait des pavillons qui ressemblaient à ceux d'EPCOT, pavillons de la Communication, du Temps, de la Terre et de l'Eau, de la Santé. Il y avait déjà celui en forme de cristal de quartz et celui du Futuroscope avec Christophe Colomb en robot (audio-animatronique) qui nous faisait voyager de l'infiniment petit à l'infiniment grand pour aboutir avec le lévitoscope au décor évoquant le coeur des galaxies. Mais dès 1988, sans que cela paraisse trop, le Futuroscope se définissait comme \&le Parc Européen de l'Image», Et c'est ce qu'il est devenu, l'endroit où l'on peut voir le plus grand rassemblement de techniques cinématographiques de pointe, Imax, Omnimax, cinémas en relief, dynamique, circulaire, showscan, et le spectacle *Les démons apprivoisés* à écrans multiples du montréalais Émile Radok, présenté à l'Expo 1986 de Vancouver. Bien sûr, Il y a la Gyrotour, le Monde des Enfants, et l'espace interactif Philips, mais on est loin de la participation généralisée du public entrevue pour le $\star$ Projet du nouveau Soleil ou Soleil levant».

Je pense que monsieur Monory avait dès le départ son idée de technopole, et qu'il l'a vendue à ses électeurs en faisant miroiter l'aire d'amusement. En 1984, c'était le début de la vague des parcs thématiques en France! On lui a reproché de parler de participation et d'interaction, mais de privilégier une relation passive du public. II répond: *Il y a deux publics, un public qu'll faut acclimater à quelque chose qu'il ne connaît pas, et un autre public qui lui se trouve déjà sensibilisé à ces nouvelles technologies. La difficulté est d'intéresser ce grand public. C'est en outre l'un des problèmes que rencontre l'homme politique: comment «vendres à des gens qui votent pour vous des choses ou des idées qu'ils ont pour l'heure peine à concevoir ou admettrom?

\section{Le développement économique}

Dans lecas présentet le *Project Sunrises, force nous est de constater qu'un parc thématique peut être gếnérateur de toutes sortes de nouvelles formes de développement économique, *Sans recourir à l'emprunt, sans avoir à augmenter les impôts, dit monsieur Monory, nous aurons invest l'an prochain» (en 1992), *un miliard de francs»... et... *une partie des bénéfices dégagés est redistribués aux communes du départemento ${ }^{(10)}$. Car le Futuroscope, c'est aussi les aires de formation et d'activités technologiques. La force de monsieur Monory est d'avoir obtenu le Téléport en 1988. France-Télécom reconnaissait le Futuroscope comme zone expérimentale et client unique, ce qui voulait dire réductions tarifaires au volume, avec la conséquence qu'une vingtaine d'entreprises étaient déjà installées sur le site en 1990. Avec en plus son Palais des congrès et sa Halle technologique, le Futuroscope se situe au coeur des activités d'échanges au niveau des technologies de pointe. Et cela est renforçé par sa zone de formation.

Dès septembre 1987, on ouvraitle *Lycéepilote innovant et Universitéw, qui donne des diplômes en Sciences et Techniques de la Communication, Droit de la Communicatique, Audio-visuel, et Sciences de l'Education par l'Audio-visuel. Plus tard est venu le Centre national d'enseignement à distance (CNED) qui peut donner des cours à peu près partout dans le monde en visio-conférence interactive, comme on avait pu le voir au pavillon INS-NTT de l’Expo 1985 de Tsukuba pour le Japon.

La plupartdes édifices du Futuroscope ont une architecture futuriste, symbolisant un nouveau rapport entre la Science et le Nouvel Age. L'Institut international de Prospective a la forme d'un lotus. Il organise des confếrences et séminaires regroupantde grands spécialistes sur la prospective des besoins humains, de même que des colloques trimestriels pour les entreprises régionales sur l'évolution des marchés. L'Institut loge aussi un club d'experts internationaux, le Club de Poitiers, qui font de la recherche fondamentale sur les modes deviedu troisième millénaire, de même que le Centre de Service $\star D$ Droit et Média».

\section{Nécessité de l'éducation permanente}

Le Futuroscope a concrétisé une grande partie de ce que nous avions rêvé pour le *Project Sunrise». *D'ici la fin du siècle, 5 millions d'emplois non qualifiés vont disparaître progressivement en Frances, dit monsieur Monory ${ }^{(11)}$. Il va y avoir une redistribution complète des temps consacrés aux loisirs, à l'éducation et au travail. Les relations avec la culture seront bouleversés. Un nouveau type ou des nouveaux types de cultures émergeront. L'éducation permanente est de plus en plus une nécessité pour comprendre le monde et nous y intégrer.L'enseignement de demain existe déa avec la télévision interactive, les vidéodisques, et petits groupes de recherche supervisés. La mise à disposition à travers les médias électroniques de connaissances variées en très grande quantité aboutit à une transformation de l'éducation: il faut avant tout montrer à l'étudiant comment chercher l'information. Et celui-ci sera évalué non plus sur la somme de ses connaissances, mais sur sa «capacité de s'exprimer avec les médias, de rechercher les 
informations, de s'organiser ${ }^{. . .}$*et de travailler en équipes, écrit Nathalie Faure dans La Vie quotidienne en $2015^{(1)}$.

Le musée et le parc d'amusement, institutions de la société industrielle, sont en crise: Tintinestau muséeet Mickey Mouse a son université. Le musée veut devenir amusant, et le parc d'amusement, éducatif? Naissent aujourd'hui les *nouveaux lieux de loisirs éducatifs $\approx$ qui assument les fonctions autrefois séparées. Le Futuroscope est un de ces lieux. Mais il fait encore la différence entre grand public et public averti. Le premier va au parc d'attractions, et le second dans l'aire de formation. Il est dans l'intérêt des technopoles d'établir un lien permanent avec legrand public. Sinon, elles risquent d'être contestées. Ce lien passe néoessairement par les *nouveaux lieux de loisirs éducatif́s\%.

\section{Les nouveaux lieux de loisirs éducatifs}

Nous avons rêvé avec le «Project Sunrise» d'un parc thématique laboratoire d'expérimentations, générant toutes sortes de types de recherches et de produits adaptés aux besoins du consommateur, générant de nouveaux modes de vie, un parc «laboratoire social» du fait de la réaction des gens aux expériences proposées. La participation active du grand public à des expériences *hands-ons était pour nous essentielle. Le grand public pour comprendrece qui se passe est comme un enfant. Il a besoin de comprendre avec son corps ce qu'il n'a pu assimiler avec sa tête, de revivre l'expérience en la jouant. Le taux de fréquentation des salons interactifs annuels *Expotecs et *Images du Futurs à Montréal est un bon indice. Et un bon nombre de gens nous disent qu'au Musće de la Villette, les parties les plus appréciées sont celles où l'on apprend par le jeu, Inventorium, Mathématiques et Univers, la section sur le cinéma, et celle où il y a les *démos* de l'Exploratorium.

Le parc thématique à l'origine est un décor pour susciter l'action chez les gens, le mélange des classes sociales au Vauxhall de Londres de 1728. Avec Disneyland, on reprend l'expérience des «scenic railways* de la fin du XIX ${ }^{e}$ siècle, c'est-à-dire l'idée devoyage à travers un décor dans un wagon qui brasse ou autre machine à vertige. Aujourd'hui les reconstitutions historiques ou historiettes fantaisistes de certains parcs thématiques semblent dépassées. La Réalité, dit-on, dépasse la Fiction! Et c'est la grande fascination. Qu'est-ce que la Réalite?

\section{L'apprentissage par le jeu}

J'ai parlé de deux parcs, *Project Sunrises et *Futuroscopes, liés à des technopoles. On peut aussi imaginer de nouveaux parcs thématiques en pleine nature, générant de nouvelles activités économiques, tourisme d'aventure, recherches et développements alternatifs en foresterie, etc. Exemple: un parc où des Amérindiens nous enseigneraient l'écologie de la terre et de l'esprit, en nous impliquant dans diverses façons de reboiser la forêt. Le travail social obligatoire est toujours refusé parce qu'imposé, le même travail, présenté sous l'angle d'un apprentissage, devient intéressant? À quoi jouent les «Rainbow Warriorss? La différence entre un centre d'interprétation et un parc thématique réellement d'aujourd'hui est qu'on y apprend par le jeu.

Les technologies de pointe nous fournissent de magnifiques outils et une nouvelle compréhension de la réalité. Mais tout cela doit être repensé dans des scénarios tenant compte des 4 grandes catégories traditionnelles de jeux et de leurs multi-croisements, hasard, habilité, vertige, et simula$\operatorname{cre}^{(13)}$, sans oublier évidemment ceux des *centres de ressourcements\% ou *groupes de croissance Nouvel Agew, en vogue dans les technopoles, jeux coopératifs, psy-jeux, rituels, méditations actives, psychodrame, thêatre archétypal, etc. $f$

\section{Notes}

(1) Alvin Toffler, vidéo de TV, Ontario, La troisiàme vague.

(2) Anita Rudman, «Des cerveaux au soleils, Le Monde, Paris, 21 févier 1987.

(3) Thierry Gaudin et associess, 2100, rbch du prochain alecle, Payot, Paris, 1990, pp. 415-420.

(4) Caroline Brun, L'lrrationnel dans Yentraprise. Balland, Paris, 1989.

(5) Claire Narbonne, Matiere grise et pins parasols, .., revue L'Expansion, Paris, no 257 , 8 mars 1985 , pp. 92-97.

(6) Jean-Pierre Garnier, Le eaphalieme high tech, Spartacus, Paris, 1988.

(7) Corine Maclaughlin et Gordon Davidson, Lee batiliseurs de l'Aube: des communautés dane un monde en transformation, Le Souffle d'Or, Paris, 1985.

(8) A propos du Futuroscope entretien avec Rend Monorym, revue Art Preas, Paris, no 12, 1991, pp. $115-118$.

(9) Idem.

(10) Idem.

(11) Idem.

(12) Nathalie Faure et Anne Michel, Des ingénieurs pedagogiques a, in La Vie quatidianne en 2015. numero hors subrie 172 de la revue sclence et Vle. Paris, septembre 1990, pp. 109-113.

(13) Roger Caillois, Les jeux et les hommes, Callimard, Paris, 1958. 